\title{
Attenuation of bleomycin induced lung fibrosis by erdosteine and inhibition of the inducible nitric oxide synthase
}

\author{
Guzel A ${ }^{1}$, Kayhan $\mathrm{S}^{2}$, Tutuncu $\mathrm{S}^{3}$, Guzel A ${ }^{4}$, Duran L $\mathrm{L}^{5}$, Alacam $\mathrm{H}^{6}$, Gunaydin $\mathrm{M}^{7}$, Caglar Torun $\mathrm{A}^{8}$, \\ Yilmaz $\mathrm{MZ}^{8}$, Selcuk MY ${ }^{9}$, Murat $\mathrm{N}^{10}$ \\ Ondokuz Mayis University, Faculty of Medicine, Department of Chest Disease, Samsun, Turkey. \\ aygul.guzel@yahoo.com
}

\begin{abstract}
Background and objectives: Despite advances in treatment modalities, the discovery of optimal medical therapies still remains a necessity in the management of pulmonary fibrosis.

Material and methods: The experiments were performed in 35 adult Sprague Dawley rats, randomly allotted into one of five groups ( $n=7)$. The control group was treated with $1 \mathrm{ml} / \mathrm{kg}, 0.9 \%$ saline; the BLM group was given a single dose of BLM (2.5 U/kg); the BLM+ER group was treated with ER (10 mg/kg/day po) for 14 days after BLM administration; the BLM+SMT group was treated with i.p injections of SMT $(20 \mathrm{mg} / \mathrm{kg} /$ day) for 14 days after BLM administration; the BLM+ER+SMT group was treated with ER and SMT for 14 days after BLM administration. At the end of day 14, the results of histopathological, biochemical, and immunohistochemical investigations were analyzed.

Results: Serum TNF- $\alpha$, nitrate/nitrite, and TBARS levels significantly increased in BLM group compared to control group ( $p<0.001, p<0.001$ and $p<0.05$ respectively). Lung tissue content of IL-6 was found to be lower in BLM+ER, BLM+SMT and BLM+ER+SMT groups compared to BLM group by immunhistochemical examinations $(p<0.01, p<0.01$ and $p<0.001$, respectively). Similarly, the TNF- $\alpha$ reactions ( $<<0.01$ for each group) and NF-kB expressions were shown to be significantly different among the study groups $(p<0.05, p<0.05$ and $p<0.001$, respectively).

Conclusion: Based on our study, ER and SMT attenuate BLM-induced pulmonary fibrosis; the combination of two agents has a greater protective efficacy against fibrosis than one alone, reducing the inflammatory markers (Tab. 2, Fig. 2, Ref. 31). Text in PDF www.elis.sk.

Key words: bleomycine, pulmonary fibrosis, erdosteine, S-methylisothiourea, rats.
\end{abstract}

\section{Introduction}

Pulmonary fibrosis is an idiopathic, progressive and lethal lung disease that can be caused by interstitial lung diseases induced by infections, connective tissue diseases, or exposure to drugs, toxins or noxious gases (1). The mechanism of pulmonary fibrosis is based

${ }^{1}$ Ondokuz Mayis University, Faculty of Medicine, Department of Chest Disease, Samsun, Turkey, ${ }^{2}$ Rize University, Faculty of Medicine, Department of Chest Disease, Rize, Turkey, ${ }^{3}$ Ondokuz Mayis University, Faculty of Veterinary Medicine, Department of Histology and Embriology, Samsun, Turkey, ${ }^{4}$ Ondokuz Mayis University, Faculty of Medicine, Department of Pediatrics, Samsun, Turkey, ${ }^{5}$ Ondokuz Mayis University, Faculty of Medicine, Department of Emergency Medicine, Samsun, Turkey, ${ }^{6}$ Ondokuz Mayis University, Faculty of Medicine, Department of Medical Biochemistry, Samsun, Turkey, ${ }^{7}$ Ondokuz Mayis University, Faculty of Medicine, Department of Pediatric Surgery, Samsun, Turkey, ${ }^{8}$ Ondokuz Mayis University, Faculty of Dentistry, Department of Pedodontics, Samsun, Turkey, ${ }^{9}$ Ondokuz Mayis University, Faculty of Medicine, Samsun, Turkey, and ${ }^{10}$ Ondokuz Mayis University, Department of Industrial Engineering, Samsun, Turkey

Address for correspondence: A. Guzel, MD, Ondokuz Mayis University, Faculty of Medicine, Department of Chest Disease, Samsun, Turkey. Phone: $+90.362 .3121919 / 4086$

Acknowledgements: This study was financially funded by Ondokuz Mayis University Scientific Research Project Management Office (Samsun, Turkey) under project number PYO.TIP.1901.12.043. on several inflammatory mediators and excessive production of fibrous connective tissues around the alveoli. Besides numerous side effects, corticosteroids and immunosuppressive agents have a limited antifibrotic effect and the results of long-term survival rates are extremely poor (1). Thus, there is a need for new drugs having better anti-fibrotic and anti-inflammatory effects for pulmonary fibrosis.

Excessive inflammatory reactions, increased oxidative stress and impaired mucoregulation are the main risk factors for development of pulmonary fibrosis The mucolytic agent erdosteine (ER) (N-carboxymethylthioacetyl, homocysteine thiolactone) is rapidly absorbed after oral administration and transformed to a biologically-active metabolite, N-thiodiglycolyl-homocysteine, which contains the sulfhydryl group that cleaves the disulphide bonds of mucin glycoproteins (2). In addition to mucoregulation, ER has anti-inflammatory and anti-oxidant properties as well as bacterial anti-adhesive effects (2). Polymorphonuclear neutrophils can generate superoxide anions and nitric oxide, ER showed a marked inhibition of the reactive oxygen species (ROS) (3). We hypothesized that ER with these mentioned mechanisms of action may protect the lungs from fibrosis after exposure to hazardous effects.

Overproduction of nitric oxide seems to be an important factor in the pathology of inflammatory processes in lung injuries and little is known about the histopathological benefits of S-methyl- 
isothiourea sulfate (SMT), a nitric oxide synthase inhibitor on the development of pulmonary fibrosis. Selective inhibition of the inducible nitric oxide synthase (iNOS) has been found effective in reducing tissue damage in several models of lung injury due to toxic exposures (4).

The animal model of BLM-induced pulmonary fibrosis has been developed to investigate the mechanisms, pathophysiology and treatment of idiopathic pulmonary fibrosis. Several inflammatory mediators such as nitrite, nitrate, thiobarbituric acid reactive substances (TBARS), interleukin-6 (IL-6), tumor necrosis factor-alpha (TNF- $\alpha$ ) and nuclear factor kappa-b (NF-kB) have been implicated in the development of lung inflammation in circulation and the contents of pulmonary tissues. In this study, we aimed at investigating the potential efficacy of ER and SMT in treating BLM-induced pulmonary fibrosis by measurements of serum nitrate/nitrite, TNF- $\alpha$ and TBARS levels and examining the lung tissue contents of IL- 6 , TNF- $\alpha$ and NF-kB by immunhistochemical staining.

\section{Material and methods}

\section{Experimental model and Study Groups}

This experimental study was approved by The Ethical Committee of Experimental Animal Studies of Samsun Ondokuz Mayis University. The rats were treated according to the guidelines of the Animal Subjects Committee of Ondokuz Mayis University. A total of 35 adult male Spraque Dawley rats from the Ondokuz Mayis University Experimental Animal Center, Samsun, Turkey, weighing between 250-300 g, were used. All rats were kept in standard experimental laboratory conditions $\left(24{ }^{\circ} \mathrm{C} ; 12 / 12 \mathrm{~h}\right.$ dark/ light cycle; free access to food and water; $60 \%$ relative humidity). The animals were divided into 5 groups ( $n=7$ per group).

The control group was treated with $1 \mathrm{ml} / \mathrm{kg}, 0.9 \%$ saline $(\mathrm{pH}$ : 5.3) by intratracheal and intraperitoneal administration. In the BLM group, a single dose of $2.5 \mathrm{U} / \mathrm{kg}$ bleomycin (Bleocin; bleomycin hydrochloride; Nippon Kayaku Co., Ltd., Tokyo, Japonya) dissolved in $0.3 \mathrm{~mL}$ sterile saline $(0.9 \% \mathrm{NaCl})$ were administered to mice by intratracheal injection on day 0 . The dose of BLM was selected as $2.5 \mathrm{U} / \mathrm{kg}$ according to a recent experimental study (5). In the BLM+ER group, oral erdosteine treatment $(10 \mathrm{mg} / \mathrm{kg} /$ day $)$ was started on day -1 , and administrated up to day 14 . The drug dose was chosen according to Erden et al.'s study (6). BLM was given by intratracheal route as $2.5 \mathrm{U} / \mathrm{kg}$ on day 0 . In the BLM+SMT group, S-methylisothiourea (SMT, $20 \mathrm{mg} / \mathrm{kg} / \mathrm{gday}$ i.p) was started on day -1 , and administrated up to day 14 (4). BLM was given by intratracheal route as $2.5 \mathrm{U} / \mathrm{kg}$ on day 0 . In the BLM+ER+SMT group, SMT (20 mg/kg/day i.p) and ER (10 mg/kg/day p.o) was started on day -1 , and administrated up to day 14 . BLM was given by intratracheal route as $2.5 \mathrm{U} / \mathrm{kg}$ on day 0 .

The rats were preoperatively anesthetized with ketamine/xylazine $(75 / 10 \mathrm{mg} / \mathrm{kg})$. The trachea was exposed through an anterior neck incision and a direct puncture with a 21 -gauge needle below the larynx was performed for instillation and aspiration procedures. At the end of the operations, the tracheal openings were sutured. After the fourteenth day, all the rats were sacrificed following an i.p. ketamine hydrochloride injection. To complete the study, the rats' lungs were removed from the thorax for histopathological and immunohistochemical investigations. Histopathological assessments were blinded and performed by an experienced pathologist.

\section{Morphological analysis and immunohistochemical staining}

The rates of pulmonary inflammation and fibrosis were analyzed by a professional histopathologist, who was blinded as to groups. After the samples of lung tissues were removed by appropriate techniques, they were fixed with $10 \%$ paraformaldehyde solution in sterile phosphate-buffered saline and embedded in paraffin wax. Sections were cut at $5 \mu$ using a microtome, and deparaffinized tissue sections were subjected to Crossmon's trichrome method and Hematoxylin-Eosin staining for histological examination.

The reactions of TNF- $\alpha$, IL- 6 and NF-kB were investigated by using immunohistochemical streptavidin-biotin-complex method in lung tissues (7). For immunohistochemical stainings, primary antibodies of rabbit policlonal IL-6 (Abcam, AB-6672), rabbit policlonal TNF- $\alpha$ (Abcam, AB-9739) and mouse policlonal NF-kB (Santa Cruz, SC-8008, CA, USA) were used. It was used LSAB+ System -HRP (Santa Cruz, SC-2051, CA, USA) for detection of secondary antibodies of IL-6 and TNF- $\alpha$, and Histostain Plus Rabbit Primary System (Zymed kit: 85-6743) for detection of NF-kB.

The sections were submitted to deparaffinization and permeabilized in a citrate buffer ( $\mathrm{pH}: 6.0$ ) solution by microwaving them at 700-watt power for proteolysis. The sections were then washed in a phosphate buffer solution (PBS) and endogenous peroxidase activities were blocked by $3 \% \mathrm{H} 2 \mathrm{O} 2$ for $15 \mathrm{~min}$ at room temperature. After washing with PBS three times, the sections were blocked with rabbit serum for $30 \mathrm{~min}$, followed by incubation with primary antibodies against the TNF- $\alpha$ antigen, (1:100), NF$\mathrm{kB}$ antigen (1:100) and IL-6 antigen (1:500) at $4{ }^{\circ} \mathrm{C}$ overnight. After being washed again with PBS, the sections were incubated in biotinylated secondary antibody for $30 \mathrm{~min}$ at $37^{\circ} \mathrm{C}$. The sections were then washed once further in PBS and incubated in streptavidin-HRP complex. Antibody binding was detected with a 3, 3'-diaminobenzidine kit, and the sections were counterstained with hematoxylin.

The intensity and distribution of positive staining in immunohistochemical examination was evaluated using a standard four-point scoring scale for intensity, with slides being scored as negatively, mildly $(+)$, moderately $(++)$, severely or strongly $(+++)$ stained (8). Histological pictures were taken using a Nikon digital-sight imaging system attached to Nikon E-600 microscope (Nikon Corporation, Japan).

\section{Biochemical evaluations}

\section{Preparation of the samples}

After sacrificing the rats, the blood samples were collected into test tubes. The whole blood was allowed to clot at room temperature. Then, these samples were centrifuged at $3000 \mathrm{x} g$ for 10 minutes. Following centrifugation, the serum samples were stored at $-80^{\circ} \mathrm{C}$ until analysis. 


\section{Measurement of serum TBARS levels}

TBARS levels were studied in line with the directions of the manufacturer (Catalogue No. 10009055, Cayman Chemical Company, USA). The results were presented in $\mu \mathrm{mol} / \mathrm{L}$.

\section{Measurement of serum nitrate/nitrite levels}

Nitrate/Nitrite levels were studied in line with the directions of the manufacturer (Catalogue No. 780001, Cayman Chemical Company, USA). This colorimetric assay provides a method for measurement of total nitrate/nitrite concentration. The results were presented in $\mu \mathrm{mol} / \mathrm{L}$.

\section{Measurement of serum TNF- $\alpha$ levels}

TNF- $\alpha$ levels were studied in line with the directions of the manufacturer (Catalogue No KRC3011, Invitrogen Corporation, USA). The principle of the test is a solid phase sandwich Enzyme Linked-Immuno-Sorbent Assay (ELISA). The results were presented in $\mathrm{pg} / \mathrm{mL}$.

\section{Statistical methods}

All data were analyzed with SPSS 21.0 package software. The measurements obtained were expressed as mean $( \pm)$ standard deviation (SD). Histopathological scores were compared with Mann-Whitney U tests. All biochemical measurements were normally distributed and analyzed with Independent-Sample $\mathrm{T}$ tests. The statistical significance level was accepted to be $\mathrm{p}<0.05$.

\section{Results}

\section{Biochemical results}

Serum TNF- $\alpha$, Nitrate/Nitrite, and TBARS levels were increased in BLM groups compared to the control group $(\mathrm{p}<0.001$, $\mathrm{p}<0.001$, and $\mathrm{p}<0.05$, respectively) (Tab. 1). After ER treatment, only serum TNF- $\alpha$ and Nitrate/Nitrite levels were statistically decreased in ER treatment groups ( $p<0.01$ and $p<0.01$ ) (Tab. 1). However, serum TNF- $\alpha$, Nitrate/Nitrite, and TBARS levels were decreased with SMT treatment $(\mathrm{p}<0.01, \mathrm{p}<0.001$, and $\mathrm{p}<0.05$, respectively) (Tab. 1). In BLM+ER+SMT group, serum TNF- $\alpha$ and Nitrate/Nitrite levels were statistically decreased $(\mathrm{p}<0.001$ and $\mathrm{p}<0.001$ ) (Tab. 1). Treatment groups were compared and the decreases in serum TNF- $\alpha$ and Nitrate/Nitrite levels were found to be statistically significant in the BLM+ER+SMT group compared to other treatment groups (Tab. 1).
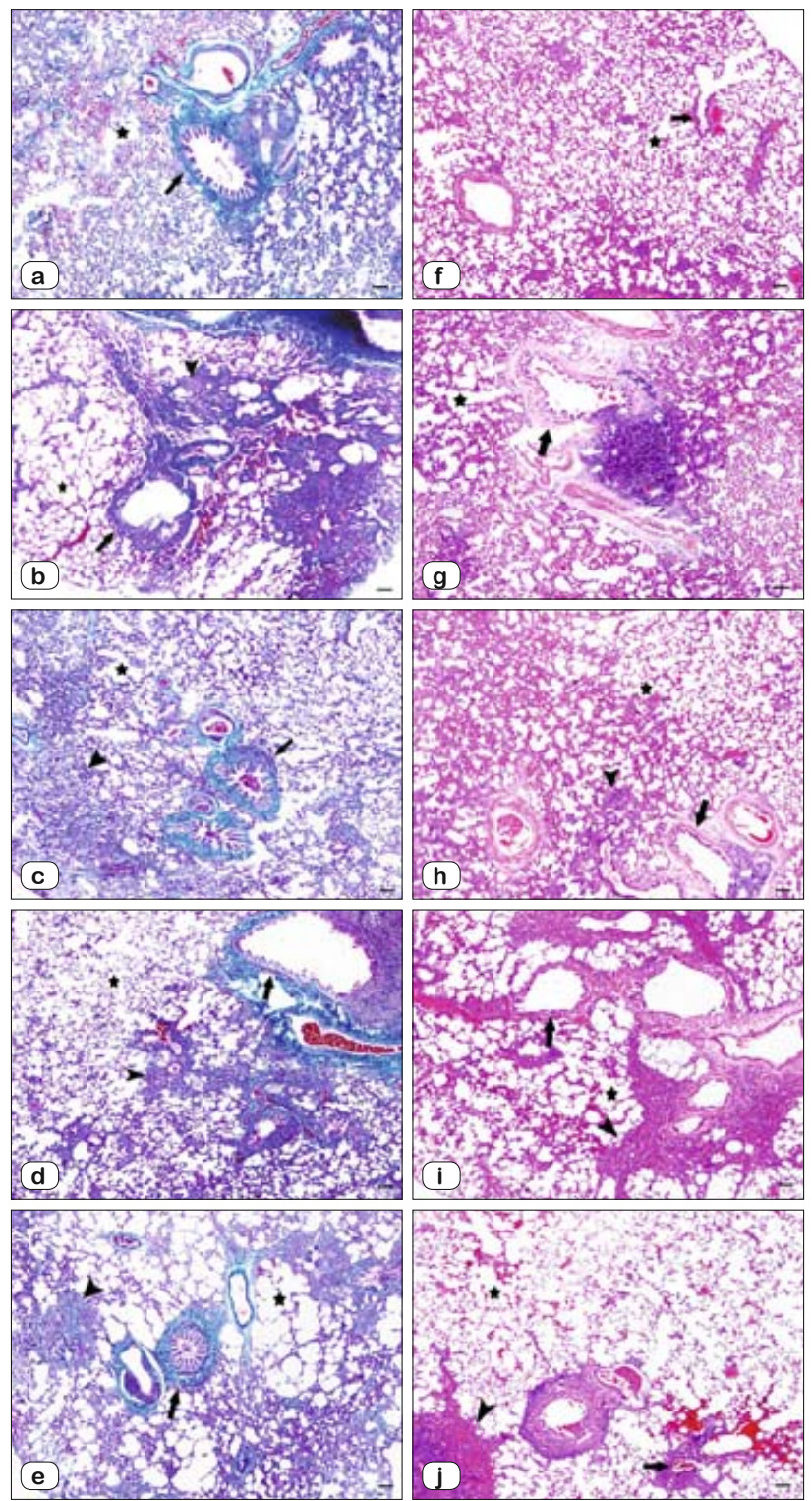

Fig. 1. Lung tissue samples were stained by Crossmon's trichrome staining of each study group (a - control group, b - BLM group, $c-$ BLM+ER group, $d-B L M+S M T$ group, $e-B L M+E R+S M T$ group) and Hematoxylin-Eosin staining of each study group (f - Control group, $g$ - BLM group, $h$ - BLM+ER group, $i-B L M+S M T$ group, $j$ - BLM+ER+SMT group). Star; alveolar space, arrow; bronchiolar epithelium, arrow head: thickenning of interalveolar septum, Bar $50 \mu \mathrm{m}$.

Tab. 1. Differences of serum TNF- $\alpha$, Nitrate/Nitrite, and TBARS levels in study groups.

\begin{tabular}{|c|c|c|c|c|c|}
\hline \multirow{2}{*}{ Biochemical parametres } & \multicolumn{5}{|c|}{ Study Groups } \\
\hline & Control & BLM & $\mathrm{BLM}+\mathrm{ER}$ & $\mathrm{BLM}+\mathrm{SMT}$ & $\mathrm{BLM}+\mathrm{ER}+\mathrm{SMT}$ \\
\hline $\mathrm{TNF}-\alpha(\mathrm{pg} / \mathrm{mL})$ & $10.05 \pm 3.27$ & $31.78 \pm 8.17^{\mathrm{a}}$ & $17.73 \pm 4.27^{\mathrm{c}}$ & $16.93 \pm 2.60^{c}$ & $11.76 \pm 4.64^{\mathrm{f}, \mathrm{g}, \mathrm{i}}$ \\
\hline Nitrate/Nitrite $(\mu \mathrm{mol} / \mathrm{L})$ & $8.13 \pm 1.70$ & $26.38 \pm 8.01^{\mathrm{a}}$ & $13.96 \pm 2.89^{\mathrm{c}}$ & $11.54 \pm 4.09^{\mathrm{d}}$ & $6.37 \pm 2.45^{\mathrm{fh}, \mathrm{i}}$ \\
\hline TBARS $(\mu \mathrm{mol} / \mathrm{ml})$ & $7.70 \pm 1.87$ & $20.69 \pm 11.77^{b}$ & $11.92 \pm 2.26$ & $8.99 \pm 1.84^{\mathrm{e}}$ & $10.21 \pm 2.47$ \\
\hline
\end{tabular}

All data with normal distribution are presented as mean \pm SD. BLM; Bleomycine-aspirated groups, BLM+ER; Bleomycine-aspirated and treated with Erdosteine groups, BLM+SMT; Bleomycine-aspirated and treated with S-Methylisothiorea sulfate groups, BLM+ER+SMT; Bleomycine-aspirated and treated with Erdosteine and S-Methylisothiorea sulfate groups, TNF- $\alpha$; Tumor Necrosis-alpha, TBARS; Thiobarbituric Acid Reactive Substances.

${ }^{a} \mathrm{p}<0.001$ compared to control group, ${ }^{b} \mathrm{p}<0.05$ compared to control group, ${ }^{\mathrm{c}} \mathrm{p}<0.01$ compared to BLM group, ${ }^{\mathrm{d}} \mathrm{p}=0.001$ compared to BLM group, ${ }^{\mathrm{e}} \mathrm{p}<0.05$ compared to BLM group, ${ }^{\mathrm{f}} \mathrm{p}<0.001$ compared to BLM group, ${ }^{\mathrm{g}} \mathrm{p}<0.05$ compared to BLM+ER group, ${ }^{\mathrm{h}} \mathrm{p}<0.001$ compared to BLM + ER group, ${ }^{\mathrm{i}} \mathrm{p}<0.05$ compared to BLM+SMT group 

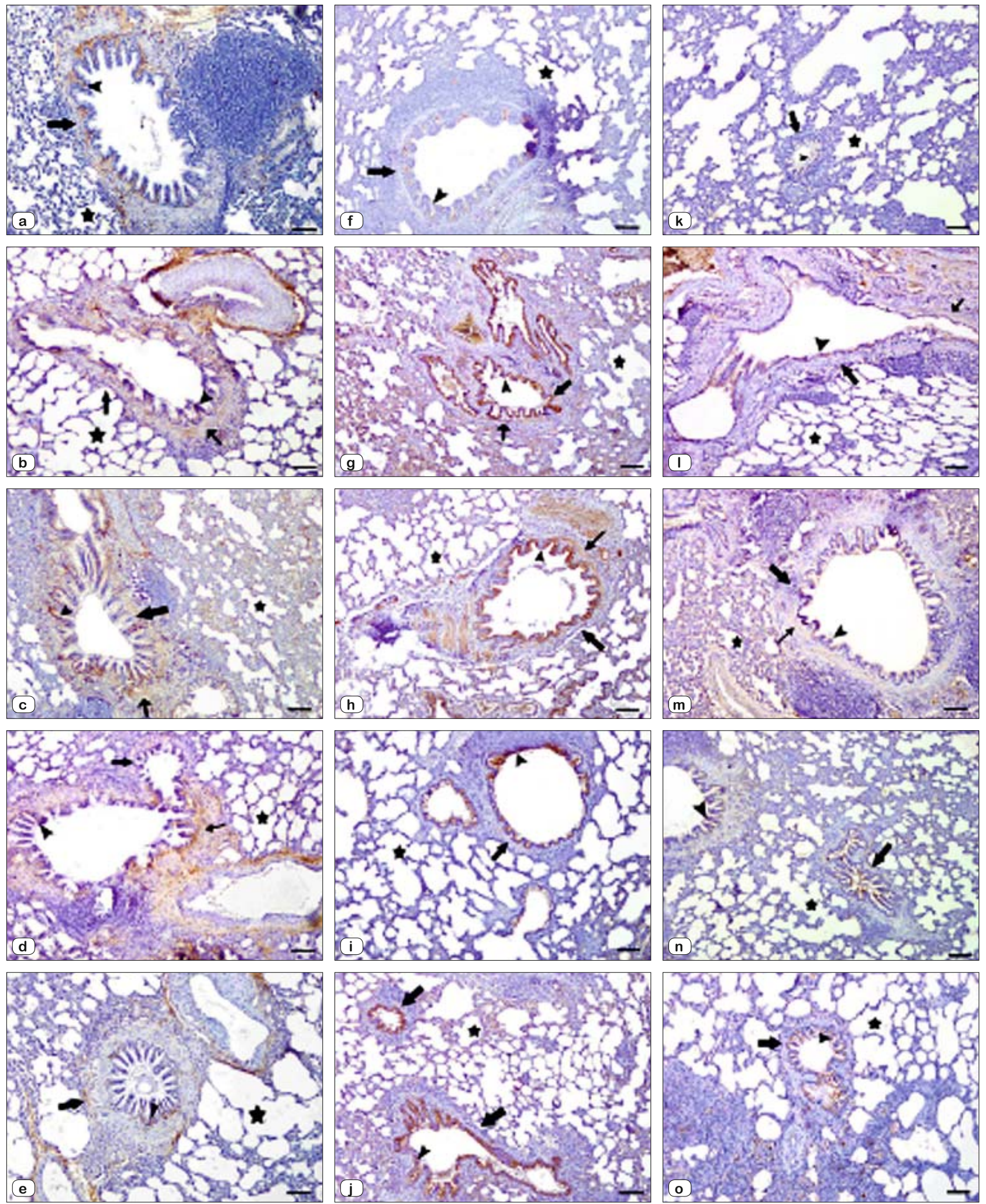

Fig. 2. Representation of lung section images of immunohistochemical staining of each study group. IL-6 (a - contol grubu, b - BLM grubu, c - ER grubu, d - SMT grubu, e - ER-SMT group), TNF- $\alpha$ (f - contol group, g - BLM group, $h-B L M+E R$ group, $i-B L M+S M T$ group, $j$ BLM+ER+SMT) and NF-kB (k - contol group, 1 - BLM group, $m$ - BLM+ER group, $n$ - BLM+SMT, o - BLM+ER+SMT). Arrow: alveolar space, large arrow: bronchiole, small arrow: smooth muscles in bronchiolar wall, arrow head: bronchiolar epithelium, Bar $50 \mu \mathrm{m}$. 


\section{Histopathological results}

The structures of bronchi, bronchioles and alveoles were examined and it was determined that there were some morphological differences between the groups. There was a mild degeneration in bronchial epithelium, mild thickening in interalveolar septum and irregular lymphocytic infiltration in the control group (Figs 1a, f). Very severe degeneration and necrosis in the bronchial and bronchiolar walls was found in the BLM group compared to the control group. Diffuse interalveolar septal thickening, accumulation of exudative fluids (edema) in alveolar space, diffuse infiltration of macrophages and lymphocytes in peribronchiolar connective tissue were the other prominent results in the BLM group (Figs $1 \mathrm{~b}, \mathrm{~g})$. In the BLM+ER group, interalveolar septal thickening of the lung tissue was decreased compared to BLM group. Interstitial thickening was not diffuse, and remained only in some local areas. Peribronchiolar lymphocytic infiltration was detected in connective tissues. The intensity of degenerations in bronchial and bronchiolar epithelium was decreased in the LEF group compared to BLM group (Figs 1c, h). Mild degenerative changes (necrosis) in bronchial walls, mild thickening of alveolar septum and lymphocytic infiltration in interstitium and alveolar space were observed in control group (Figs 1c, f). The histopathologic findings were found to be similar with BLM+SMT and BLM+ER groups (Figs $1 d, i)$. In the BLM+ER+SMT group there was a prominent decrease in thickening of interalveolar septum compared to BLM+ER and BLM+SMT groups (Figs 1e, j).

\section{Immunohistochemical results}

The details of immunohistochemical reactions and comparison of groups are shown in Table 2. No immunohistochemical reaction of IL-6 in interalveolar septum was found in the control group. A weak IL-6 reaction was determined in peribronchial and vascular epithelium (Fig. 2a). In the BLM group, immunohistochemical staining of IL-6 was strongly positive in alveolar septal regions. There were strong positive IL-6 reactions in muscle cells surrounding the bronchi and bronchioles, periadventitial connective tissues of vessels and epithelium of bronchi and bronchioles and alveolar macrophages (Fig. 2b). In both BLM+ER and BLM+SMT groups, a dense lymphocytic infiltration and strong positive IL-6 reaction were observed in thickened septal areas (Fig. 2c, d). In the BLM+ER+SMT group, a weak immune reaction of IL- 6 was found in bronchiolar epithelium, interalveolar septum and peripheral regions of vessels compared to BLM+ER and BLM+SMT groups (Fig. 2e).
In the control group, there was a mild degree of immune positive reactions by TNF- $\alpha$ in some of bronchiolar epithelium of lung tissues (Fig. 2f). In the BLM group, a strong degree of positive TNF- $\alpha$ reaction was found where the bronchiolar and bronchial septum thickened, and peribronchiolar area was infiltrated by lymphocytes (Fig. 2g). In BLM+ER and BLM+SMT groups, a moderate degree of positive staining by TNF- $\alpha$ was determined in thickened bronchial and bronchiolar epithelium and peripheral smooth muscle cells (Figs 2h, i). In BLM+ER+SMT group, a mild degree of positive staining of TNF- $\alpha$ was found in thickened bronchial and bronchiolar epithelium and peripheral smooth muscle cells (Fig. 2j).

In the control group, there was a light immune positive reaction of NF-kB in some of bronchiolar epithelium of lung tissues (Fig. $2 \mathrm{k})$. In the BLM group, a moderate degree of positive immunohistochemical reaction of NF-kB was found where the bronchiolar and bronchial septum thickened, and peribronchiolar area was infiltrated by lymphocytes (Fig. 21). In each of BLM+ER, BLM+SMT and BLM+ER+SMT groups, a mild degree of positive staining by NF-kB was determined in bronchial and bronchiolar epithelium, as well as in some of the interalveolar septal regions (Figs 2m, n, o).

\section{Discussion}

Previous studies showed that the animal model of experimental pulmonary fibrosis generated by BLM exactly duplicates human idiopathic pulmonary fibrosis (9). In this experimental study, we successfully used this model of pulmonary fibrosis to document changes in interalveolar septal thickening, exudation in alveolar space, and diffuse infiltration of inflammatory cells in lung paranchyma.

Previous clinical and experimental studies have reported that gender may play an important role in pulmonary fibrosis (10-11). Voltz et al reported that male sex hormones exacerbate lung function impairment after bleomycin-induced pulmonary fibrosis (11). We likewise performed our study using a total of 35 adult male Spraque Dawley rats and achieved a successful experimental lung fibrosis by BLM with the dose of $2.5 \mathrm{U} / \mathrm{kg}$ without mortality.

The results of our study show that ER and SMT treatment may play an important role in the course of pulmonary fibrosis with effects as follows:

(a) Serum biochemical analysis showed that a single use of ER and SMT separately decreases the serum TNF- $\alpha$, Nitrate/Nitrite, and TBARS levels;

Tab. 2. Comparison of semiquantative immunohistochemical staining scores of IL-6, TNF- $\alpha$, and NF-Kb in study groups.

\begin{tabular}{|c|c|c|c|c|c|}
\hline & \multicolumn{5}{|c|}{ Study Groups } \\
\hline & Control & BLM & $\mathrm{BLM}+\mathrm{ER}$ & BLM+SMT & $\mathrm{BLM}+\mathrm{ER}+\mathrm{SMT}$ \\
\hline IL-6 & \pm & $+++^{a}$ & $++^{\mathrm{c}}$ & $++^{c}$ & $+e, f, g$ \\
\hline TNF- $\alpha$ & \pm & $+++^{a}$ & $++^{\mathrm{c}}$ & $++^{\mathrm{c}}$ & $++^{\mathrm{c}}$ \\
\hline NF-kB & \pm & $++^{b}$ & $+^{\mathrm{d}}$ & $+^{\mathrm{d}}$ & $\pm^{\mathrm{e}}$ \\
\hline
\end{tabular}

All findings are represented as median (semiquantitatively). BLM; Bleomycine-aspirated groups, BLM+ER; Bleomycine-aspirated and treated with Erdosteine groups, BLM+SMT; Bleomycine-aspirated and treated with S-Methylisothiorea sulfate groups, BLM+ER+SMT; Bleomycine-aspirated and treated with Erdosteine and S-Methylisothiorea sulfate groups, IL-6; Interleukin-6, TNF- $\alpha$; Tumor Necrosis-alpha, NF-Kb; Nuclear Factor Kappa b.

${ }^{a} \mathrm{p}<0.001$ compared to control group, ${ }^{\mathrm{b}} \mathrm{p}<0.01$ compared to BLM group, ${ }^{\mathrm{c}} \mathrm{p}<0.01$ compared to BLM group, ${ }^{\mathrm{d}} \mathrm{p}<0.05$ compared to BLM group, ${ }^{\mathrm{e}} \mathrm{p}<0.001$ compared to BLM group, ${ }^{\mathrm{f}} \mathrm{p}<0.05$ compared to BLM+ER group, ${ }^{\mathrm{g}} \mathrm{p}<0.05$ compared to BLM+SMT group 
(b) Combined therapy with ER and SMT was found to lower the serum levels of nitrate/nitrite and TNF- $\alpha$ compared to the single effect of each drug;

(c) ER and SMT reduce the production of cytokines IL-6, TNF- $\alpha$, and NF-kB;

(d) In ER+ SMT group, immunohistochemical staining by IL-6 showed a weaker reaction than in ER and SMT groups; and

(e) ER and SMT reduce histopathological findings such as the number of inflammatory cells, interalveolar septal thickening, and intensity of degenerations in bronchiolar epithelium.

These findings showed that both ER and SMT attenuate the BLM-induced pulmonary fibrosis, and that combining these therapies has greater protective efficacy against the fibrosis and reduces inflammatory markers to a greater extent when compared to the use of either drug on its own.

Pulmonary fibrosis due to hypoxemia-induced systemic effects is a significant issue for investigation in terms of its mechanisms and treatment modalities. Nonetheless, its exact mechanism is yet unknown. Many studies have been done on changes causing lung damage at the cellular level (12-14). It is reported that the damage caused by reactive oxygen and nitrogen species, whose release is increased by activated inflammatory cells, causing initiation of lipid peroxidation, hydroxylation, and nitrifiying amino acids, has an important role in the pathogeny of lung fibrosis (15). Treatment options should therefore be planned by considering these cellular changes demonstrated by various researchers.

After being metabolized in the liver, ER engages in antioxidant activity via the allocated sulfhydryl group protecting the cell from oxidative damage by entering the cell and increasing the production of glutathione (16-17). Because of these characteristics, ER's activity in many diseases has been the subject of research (18-20). Erden et al studied the effectiveness of ER's in a pulmonary fibrosis model, using bleomycin in rats, and demonstrated that it prevents neutrophil accumulation with inhibition by inhibiting acute lung injury and fibrosis, lipid peroxidation and chemokine production (6). Similarly, in an experimental animal study, researchers have reported an otoprotective effect of ER in ototoxicity, which they produced by using cisplatin (20).

Recently, the important role of reactive nitrogen species and peroxynitrite in the respiratory tract in the pathogenesis of lung diseases has frequently been noted (21-22). Superoxide anion, a strong ROS increase in the environment as a result of the onset of inflammation, reacts with nitric oxide to form peroxynitrite, a stronger oxidant. The resulting peroxynitrite causes tissue damage by disrupting the function of protein and nitrifying the tyrosine residues (23). Therefore, the inhibition of iNOS, which is responsible for the synthesis of nitric oxide, may be a therapeutic option for preventing pulmonary fibrosis. SMT is a relatively selective iNOS inhibitor and its therapeutic efficacy has been demonstrated in many animal studies (24-25). Guo et al treated mice with induced herpes simplex encephalitis with SMT, and reported that that was just due to inhibition of pro-inflammatory cytokines such as TNF- $\alpha$, IL-1 $\beta$, NO (26). In another study, in which they formed different models of pulmonary aspiration in rats, it was reported that SMT demonstrated a therapeutic efficacy by reducing the production of iNOS (4). To our knowledge, there is no study in literature demonstrating the efficacy of combined SMT and ER treatment in pulmonary fibrosis. Erdosteine treatment of lung injuries is often investigated by evaluating its efficacy according to other treatment modalities. In particular, the superiority of ER therapy to other treatment modalities having antioxidant and antiinflammatory properties, such as $\mathrm{N}$-acetylcysteine and Vitamin $\mathrm{E}$, was investigated (27-28). In this study, the efficacy of combining ER and SMT was evaluated and we proved the efficacy of their combination on its components.

The inflammatory mediators TNF- $\alpha$, NF-kB and IL- 6 are produced by macrophages and other inflammatory cells, and play an important role in both the initiation and progression of pulmonary fibrosis. These markers have been used in many studies to analyze the degree of inflammation and fibrosis of the lungs and correlate them with histopathological evaluations (29-31). We investigated the efficacy of ER and SMT in BLM-induced pulmonary fibrosis by measurements of serum nitrate/nitrite, TNF- $\alpha$ and TBARS levels and immunohistochemical examination of IL-6, TNF- $\alpha$ and NF-kB in lung tissue samples. The oxidative tissue damage was found to be more severe in acute phases of BLM administration, and we found a significant increase in serum TBARS levels as a marker of oxidative stress in the BLM group compared to ER- and SMT-treated groups due to a decrease in oxidative stress. Our results also showed a correlation between serum TNF- $\alpha$, nitrate, nitrite, and TBARS levels, in addition to lung tissue contents of IL-6, TNF- $\alpha$ and NF-kB, with the severity of inflammation and fibrosis in BLMinduced pulmonary fibrosis. The treatment groups were evaluated by these markers, especially as immunohistochemical examinations revealed that ER and SMT have protective effects against the inflammation and fibrosis.

As a result, our findings demonstrated that ER and SMT, particularly as combined therapy, inhibit pulmonary inflammation, decrease IL-6, TNF- $\alpha$ and NF-kB production and attenuate BLM-induced pulmonary fibrosis. These two agents merit further investigation in patients with pulmonary fibrosis to treat this lifethreatening disease.

\section{References}

1. Selman M, King TE, Pardo A. Idiopathic pulmonary fibrosis: prevailing and evolving hypotheses about its pathogenesis and implications for therapy. Ann Intern Med 2001; 134 (2): 136-151.

2. Marchioni CF, Moretti M, Muratori M et al. Effects of erdosteine on sputum biochemical and rheologic properties: pharmacokinetics in chronic obstructive lung disease. Lung 1990; 168 (5): 285-293.

3. Dal Sasso M, Culici M, Bianchi T, Fonti E, Braga PC. Inhibitory effects of metabolite 1 of erdosteine on the generation of nitric oxide and peroxynitrile chemiluminescence by human neotrophils. Pharmacology 2004; 71 (3): 120-127.

4. Guzel A, Basaran UN, Aksu B et al. Protective effects of S-methylisothiourea sulfate on different aspiration materials-induced lung injury in rats. Int J Pediatr Otorhinolaryngol 2008; 72 (8): 1241-1250. 
5. Chitra P, Saiprasad G, Manikandan R, Sudhandiran G. Berberine attenuates bleomycin induced pulmonary toxicity and fibrosis via suppressing NF- $\kappa B$ dependant TGF- $\beta$ activation: a biphasic experimental study. Toxicol Lett 2013; 219 (2): 178-193.

6. Erden ES, Kirkil G, Deveci F et al. Effects of erdosteine on inflammation and fibrosis in rats with pulmonary fibrosis induced by bleomycin. Tuberk Toraks 2008; 56 (2): 127-138.

7. True LD. Principles of immunohistochemistry. 16-22. In: True LD (Eds). Atlas of Diagnostic Immunohistopathologhy. NewYork; Gower Medical Publishing, 1990.

8. Adams EJ, Gren JA, Clark AH, Youngson JH. Comparison of Different Scoring Systems for Immunohistochemical Staining. J Clin Pathol 1999; 52 (1): 75-77.

9. Moore BB, Hogaboam CM. Murine models of pulmonary fibrosis. Am J Physiol Lung Cell Mol Physiol 2008: 294 (2); L152-160.

10. Gharaee-Kermani M, Hatano K, Nozaki Y, Phan SH. Gender based differences in bleomycin induced pulmonary fibrosis. Am J Pathol 2005; 166 (6): 1593-1606.

11. Voltz JW, Card JW, Carey MA et al. Male sex hormones exacerbate lung function impairment after bleomycin-induced pulmonary fibrosis. Am J Respir Cell Mol Biol 2008; 39 (1): 45-52.

12. Kim JY, Lee KH, Lee BK, Ro JY. Peroxynitrite modulates release of inflammatory mediators from guinea pig lung mast cells activated by antigen-antibody reaction. Int Arch Allergy Immunol 2005; 137 (2): 104-114.

13. Fubini B, Hubbard A. Reactive oxygen species (ROS) and reactive nitrogen species (RNS) generation by silica in inflammation and fibrosis. Free Radic Biol Med 2003; 34 (12): 1507-1516.

14. Alhamad EH, Cal JG, Shakoor Z, Almogren A, Alboukai AA. Cytokine gene polymorphisms and serum cytokine levels in patients with idiopathic pulmonary fibrosis. BMC Med Genet 2013; 14 (1): 66.

15. Inghilleri $\mathbf{S}$, Morbini $\mathbf{P}, \mathbf{C a m p o} I$ et al. Factors influencing oxidative imbalance in pulmonary fibrosis: an immunohistochemical study. Pulm Med 2011; 2011: 421409.

16. Sogut S, Ozyurt H, Armutcu $F$ et al. Protection of erdosteine on bleomycine-induced lung fibrosis in rats. Eur J Pharmacol 2004; 494 (23): $213-220$.

17. Yildirim Z, Turkoz Y, Kotuk $M$ et al. Effects of aminoguanidine and antioxidant erdosteine on bleomycin induced lung fibrosis in rats. Nitric Oxide 2004; 11 (2): 156-164.

18. Briguori C, Condorelli G. Effect of erdosteine on cyclosporine induced chronic nephrotoxicity. Nephrourol Mon 2012; 4 (2): 487-488.
19. Marabini L, Calò R, Braga PC. Protective effect of erdosteine metabolite I against hydrogen peroxide-induced oxidative DNA-damage in lung epithelial cells. Arzneimittelforschung 2011; 61 (12): 700-706.

20. Waissbluth S, Dupuis I, Daniel SJ. Protective effect of erdosteine against cisplatin-induced ototoxicity in a guinea pig model. Otolaryngol Head Neck Surg 2012; 146 (4): 627-632.

21. Malaviya R, Venosa A, Hall $L$ et al. Attenuation of acute nitrogen mustard-induced lung injury, inflammation and fibrogenesis by a nitric oxide synthase inhibitor. Toxicol Appl Pharmacol 2012; 265 (3): 279-291.

22. Liu L, Lu W, Ma Z, Li Z. Oxymatrine attenuates bleomycin-induced pulmonary fibrosis in mice via the inhibition of inducible nitric oxide synthase expression and the TGF- $\beta$ /Smad signaling pathway. Int J Mol Med 2012; 29 (5): 815-822.

23. Saleh D, Barnes PJ, Giaid A. Increased production of the potent oxidant peroxynitrite in the lungs of patients with idiopathic pulmonary fibrosis. Am J Respir Crit Care Med 1997; 155 (5): 1763-1769.

24. Kunak ZI, Macit E, Yaren $\mathbf{H}$ et al. Protective effects of melatonin and S-methylisothiourea on mechlorethamine induced nephrotoxicity. J Surg Res 2012; 175 (1): e17-23.

25. ArunaDevi R, Ramteke VD, Kumar S et al. Neuroprotective effect of s-methylisothiourea in transient focal cerebral ischemia in rat. Nitric Oxide. 2010; 22 (1): 1-10.

26. Guo YJ, Li W, Li XF, et al. S-methylisothiourea induces apoptosis of herpes simplex virus-1-infected microglial cells. Inflammation 2011; 34 (5): 388-401.

27. Yildirim Z, Kotuk M, Iraz M et al. Attenuation of bleomycin-induced lung fibrosis by oral sulfhydryl containing antioxidants in rats: erdosteine and N-acetylcysteine. Pulm Pharmacol Ther 2005; 18 (5): 367-373.

28. Demiralay R, Gürsan N, Erdem H. The effects of erdosteine, Nacetylcysteine, and vitamin $\mathrm{E}$ on nicotine-induced apoptosis of pulmonary cells. Toxicology 2006; 219 (1-3): 197-207.

29. Kayhan S, Guzel A, Duran L et al. Effects of leflunomide on inflamation and fibrosis in bleomycine induced pulmonary fibrosis in wistar albino rats. J Thorac Dis 2013 Sep 24. doi: 10.3978/ j.issn.2072-1439.2013.09.20.

30. Bringardner BD, Baran CP, Eubank TD, Marsh CB. The role of inflammation in the pathogenesis of idiopathic pulmonary fibrosis. Antioxid Redox Signal 2008; 10 (2): 287-301.

31. Hasegawa M, Fujimoto M, Kikuchi K, Takehara K. Elevated serum tumor necrosis factor-alpha levels in patients with systemic sclerosis: association with pulmonary fibrosis. J Rheumatol 1997; 24 (4): 663-665.

Received October 11, 2013. Accepted October 24, 2013. 